\title{
Concurrent pulmonary Mycobacterium avium complex (MAC) infection and active Hürthle cell thyroid carcinoma: is there a connection?
}

\author{
Kara M. Meinke Baehr, Whitney S. Goldner* \\ University of Nebraska Medical Center, Department of Internal Medicine-Diabetes, Endocrinology, and Metabolism, Omaha, USA \\ *Corresponding Author: wgoldner@unmc.edu
}

Received 5 October 2009; revised 5 January 2010; accepted 8 January 2010.

\begin{abstract}
We present two cases of pulmonary MAC infection in women with Hürthle cell thyroid carcinoma. Both cases were asymptomatic octogenarian women with active Hürthle cell thyroid carcinoma and prolonged periods of hypothyroidism prior to diagnosis of pulmonary MAC. Mycobacterium avium complex has never been reported in association with any type of thyroid cancer, specifically Hürthle cell carcinoma. A review of the literature and possible associations between the two are discussed in this article.
\end{abstract}

Keywords: Hürthle Cell Thyroid Cancer; Hypothyroidism; Mycobacterium Avium Complex; Cellular Immunity

\section{INTRODUCTION}

Hürthle cell thyroid carcinomas are a type of well-differentiated thyroid carcinoma and are either classified as a subset of follicular carcinoma or a separate category of well-differentiated thyroid cancer [1]. Hürthle cell carcinomas usually produce thyroglobulin, but are often less iodine avid, and are more aggressive than the other types of well-differentiated thyroid carcinoma such as papillary or follicular [2,3]. There is a female predominance with peak ages of incidence in the seventh and eighth decades [2]. Specific treatment is controversial, but generally total thyroidectomy and remnant ablation is recommended [1]. Follow-up for Hürthle cell thyroid cancer is similar to papillary and follicular thyroid cancers, which entails anatomic imaging of the neck and chest. This is usually done with neck ultrasound and computed tomography (CT) scans of the chest and occasionally neck; and when indicated, TSH-stimulated I-131 whole body scan is performed.
Laboratory follow- up includes tumor marker evaluation with serum thyroglobulin (Tg) and antibodies. Thyroid hormone suppression is also a mainstay of therapy. Despite its more aggressive course, there are no known associations between Hürthle cell thyroid cancer and immunocompromised states; specifically, there are no published reports of Hürthle cell thyroid carcinoma and pulmonary MAC infection.

MAC refers to mycobacterium infections caused by free-living nontuberculous organisms that are inhaled or ingested from the environment [4]. Among nontuberculous species, MAC is the most common cause of pulmonary disease in the United States. Diagnosis of pulmonary MAC requires both imaging consistent with pulmonary disease and microbiologic confirmation in a symptomatic patient to necessitate treatment [4]. Increased rates of MAC in immunocompromised patients with AIDS were reported in the 1990s; however, with improved anti-retroviral therapies, MAC infections in HIV infected patients have been declining [5]. The increased susceptibility of patients with AIDS to disseminated nontuberculous mycobacteria infections helps provide insight into the pathogenesis of these infections in non-immunocompromised hosts. Disseminated MAC infections usually occur when the CD4+ T-lymphocyte count is $<50 \mu$, suggesting that some intrinsic activity of the T-lymphocytes is important for immunity to mycobacteria [6]. We present two cases of pulmonary MAC infection in octogenarian women with active Hürthle cell thyroid carcinoma.

\section{CASE 1}

Case 1 is a 79 year-old female initially diagnosed with stage 2 Hürthle cell carcinoma (pathologic stage T2NOM0) in February 1999. She underwent total thyroidectomy and remnant ablation with $100 \mathrm{mCi}$ of I-131. Posttreatment scan showed uptake in the central neck consistent with thyroid remnant without evidence of local or distant 
metastases. She had no follow-up until 2006 when she presented to our institution for evaluation of multiple pulmonary nodules. Excisional biopsy of one of the lesions was consistent with metastatic Hürthle cell carcinoma. Thyroid ultrasound showed no evidence of thyroid cancer in the neck. Thyroglobulin (Tg) was 136 $\mathrm{ng} / \mathrm{mL}$ (goal $<0.1 \mathrm{ng} / \mathrm{ml}$ in remission) with negative antithyroglobulin antibodies, and thyroid stimulating hormone (TSH) of $11.5 \mathrm{mcIU} / \mathrm{mL}$. I-123 whole body scan showed uptake in the thyroid bed and superior mediastinum and stimulated $\mathrm{Tg}$ was $155 \mathrm{ng} / \mathrm{mL}$. She underwent repeat radioactive iodine ablation with $206 \mathrm{mCi}$ of I-131 using dosimetry to calculate her dose. Her posttreatment scan showed heterogeneous uptake throughout the lungs, most intense in the left lower lobe, and uptake in the thyroid bed.

Despite successful uptake on radioactive iodine scans, six months after treatment, $\mathrm{Tg}$ had risen to $341 \mathrm{ng} / \mathrm{mL}$. Repeat chest CT was unchanged. PET scan showed uptake in the right middle lobe pulmonary nodule and bilateral lower lobe lesions that were calcified with lower SUVs suggesting more benign disease. Since most of the pulmonary lesions were not PET avid, indicating they were likely radioiodine avid, she received another 249.6 $\mathrm{mCi}$ of I-131 with dosimetry in March 2008. Posttreatment scan showed faint uptake throughout the lungs bilaterally, but no uptake to correspond with the largest pulmonary nodules, suggesting the pulmonary metastases were no longer radioiodine avid. Following treatment, $\mathrm{Tg}$ continued to rise and was $3976 \mathrm{ng} / \mathrm{mL}$ in July 2008 on thyroid hormone suppression. Follow-up chest CT showed numerous pulmonary nodules, two lesions increased in size in the right upper lobe, along with scattered tree-in-bud opacities, bronchial wall thickening, and bronchiectasis suggesting mycobacterium avium complex (MAC). Induced sputum was culture positive for MAC. The patient was referred to infectious disease and no treatment was recommended since she was asymptomatic.

A PET scan showed disease progression within the mediastinum and right hilum with a maximum SUV of 12.4 in a subcarinal mass and multiple PET avid pulmonary nodules. The hilar mass was felt to be non-resectable, so she was treated with external beam radiation therapy for her mediastinal mass and sensitizing chemotherapy with doxorubicin in November 2008. After starting chemotherapy, she developed worsening productive cough, chills, and fatigue and significant oxygen desaturation. Since MAC was still present in her sputum, she was started on triple antibiotic therapy (azithromycin $500 \mathrm{mg}$ daily, rifampin $300 \mathrm{mg}$ daily, and ethambutol $800 \mathrm{mg}$ daily) for her symptomatic MAC in January 2009 . She has symptomatically improved and no longer has a productive cough; however, continues to require supplemental oxygen. Her most recent $\mathrm{Tg}$ was $1582 \mathrm{ng} / \mathrm{ml}$ (down from maximum $3976 \mathrm{ng} / \mathrm{ml}$ ) with negative antibodies and a TSH of $0.45 \mathrm{mcIU} / \mathrm{ml}$. Chest CT shows interval decrease in bilateral consolidations, unchanged bilateral bronchiectasis and 2 right lower lobe nodules, and a decrease in the subcarinal lymph node size.

\section{CASE 2}

Case 2 is an 82 year-old female diagnosed with stage 2 Hürthle cell carcinoma (T3N0M0) in July 2004 and underwent total thyroidectomy and remnant ablation with $45.8 \mathrm{mCi}$ of I-131. Post-treatment scan showed only uptake in the thyroid bed. In September 2005, she underwent a TSH-stimulated (with thyrogen) whole body scan that showed $1.6 \%$ minimal uptake in the area of the thyroid bed. Tg stimulated to $0.2 \mathrm{ng} / \mathrm{ml}$ with a TSH of $315 \mathrm{mcIU} / \mathrm{mL}$. She refused additional radioactive iodine therapy due to severe sialoadenitis with the first treatment. Thyroid ultrasound revealed residual thyroid tissue in the right bed measuring $1.2 \times 0.7 \times 1.1 \mathrm{~cm}$ and no evidence of focal nodules or lymphadenopathy.

Repeat thyroid ultrasounds in September 2006 and August 2007 showed no evidence of recurrence. Tg level ranged $0.3-0.8 \mathrm{ng} / \mathrm{ml}$, antibodies remained negative, and TSH ranged from undetectable to $3.6 \mathrm{mcIU} / \mathrm{ml}$ during this time. $\mathrm{Tg}$ increased to $2.9 \mathrm{ng} / \mathrm{mL}$ in March 2008 with an elevated TSH of $31.5 \mathrm{mcIU} / \mathrm{ml}$; however, decreased to $1.7 \mathrm{ng} / \mathrm{mL}$ in August 2008 (TSH $6.9 \mathrm{mcIU} / \mathrm{ml}$ ) and further to $1.5 \mathrm{ng} / \mathrm{mL}$ with a TSH of $0.14 \mathrm{mcIU} / \mathrm{ml}$ one month later. Thyroid ultrasound in August 2008 showed minimal right-sided residual thyroid tissue measuring $0.4 \times 0.6 \times 0.7 \mathrm{~cm}$. Non-contrast chest CT showed a right upper lobe semi-solid nodule measuring $1 \mathrm{~cm}$ that was suspicious for cancer. CT also revealed bronchiectasis changes in anterior portions suspicious for MAC; and her induced sputum was MAC culture positive. PET scan was normal. After infectious disease consultation, she was not treated for her MAC because she was asymptomatic.

Follow-up chest CT in February 2009 showed stable mixed solid and ground glass nodules in the right upper lobe, bilateral patchy consolidations, and an enlarging precarinal lymph node. This lymph node was biopsied and pathology was negative for malignancy. Tg has continued to increase and is currently $8.4 \mathrm{ng} / \mathrm{ml}$ in August 2009 , and TSH is $0.087 \mathrm{mcIU} / \mathrm{ml}$. She is still asymptomatic.

\section{DISCUSSION}

These cases are the first to report pulmonary MAC infection in persons with active Hürthle cell thyroid carcinoma. Interestingly, both these patients were asymptomatic, octogenarian women and diagnosis was initially suggested by radiographic findings and later confirmed 
with culture positive induced sputum. Typical radiologic findings consistent with pulmonary MAC infection on chest radiographs or computed tomography (CT) include nodular infiltrates, multifocal bronchiectasis, cavitation, and multiple small nodules [7]. The nodules and bronchiectasis are usually present within the same lobe and occur most frequently in the right middle lobe and lingula $[8,9]$. The keys to our patient's diagnoses were the presence of new, non-dependent bronchiectasis that was different from the other pulmonary disease previously seen on past CT scans.

Four different clinical syndromes of pulmonary MAC infection have been described. These include classic cavitary disease seen commonly in middle-aged smoking males [6]; nonclassic forms such as bronchiectasis and multiple nodules found in elderly women $[6,8]$, MAC lung disease in cystic fibrosis patients [10], and a hypersensitivity pneumonitis associated with hot tub use [11]. The clinical syndrome that is most likely in our two cases includes the group who presents with mid-lung nodular bronchiectasis with multiple nodules and tree-in-bud opacities due to MAC with no underlying lung disease or immunosuppression. This bronchiectatic form of the disease is frequently termed the "Lady Windermere syndrome" and presents in slender, middle-aged to elderly Caucasian women and involves the right middle lobe and/or lingula $[6,12]$. The first report of this form of MAC cites 21 such patients, most non-smoking women with a mean age of 66 years, who presented with symptoms of persistent cough and purulent sputum [13]. This group is homogeneous, consisting of older, Caucasian, otherwise healthy women, raising the possibility of a host defense immune defect to explain disease susceptibility [14]. Whereas mutations in the interferon-gamma and interleukin-12 production and response pathway have been proposed, no consistent immune phenotype in pulmonary nontuberculous mycobacteria infection has been clearly established [15]. Both of our patients were Caucasian women in their late 70's and early 80's with Hürthle cell thyroid carcinoma and no obvious form of immunosuppression; however, they were both asymptomatic which is in contrast to how the Lady Windermere syndrome group usually present. There are no studies linking thyroid cancer or its treatment with immunosuppression. However, Hürthle cell thyroid carcinoma often has a more aggressive course than papillary or follicular thyroid carcinoma [12] and tends to occur more frequently in older women [2]. Case 1 had pulmonary disease from her metastatic Hürthle cell carcinoma, and case 2 had a pulmonary nodule, not definitively metastatic thyroid cancer, so neither of the patients was completely devoid of lung disease as described in the Lady Windermere form of MAC pulmonary infection.

Another common feature for both cases was a prolonged period of hypothyroidism evidenced by an increased TSH prior to diagnosis of MAC. Case 1 was iatrogenically hypothyroid prior to both treatments with radioactive iodine ablation using dosimetry in April 2007 and March 2008. Case 2 was hypothyroid due to medication non-adherence from March through August 2008 which immediately preceded the diagnosis of pulmonary MAC infection on chest CT. Animal studies have shown a decrease in lymphocyte function during hypothyroidism, with a return of normal lymphocyte function during euthyroid states [16]. The number of peripheral white blood cells, mainly lymphocytes, in hypothyroid (thyroidectomized) animals is reduced to half of normal levels [17]. Other studies have shown dysregulation of CD4+ T lymphocyte responsiveness in hypothyroid dogs suggesting a relationship between hypothyroidism and cellular immunity [18]. Unfortunately specific studies regarding cellular immunity in hypothyroid humans are lacking. Radioactive iodine treatment can cause bone marrow suppression after treatment; however, this happens in all types of thyroid cancer, not exclusively Hürthle cell carcinoma, making it a less likely etiology [19]. Given both women experienced prolonged hypothyroidism immediately prior to their diagnosis of MAC, we propose that the prolonged hypothyroidism may have predisposed to suppression of cell-mediated immunity in an already vulnerable host (older, Caucasian women with potential lung pathology). This may have resulted in both patients having an increased susceptibility to acquire pulmonary MAC infection. There are no reports of altered immune function associated with any thyroid carcinomas, and specifically none that associate Hürthle cell thyroid carcinoma with altered immune function; however, this is also a potential mechanism, especially given Hürthle cell thyroid carcinoma often has a more aggressive course, and should be considered and evaluated further. Also, radiologic detection of MAC may be more common in patients with more aggressive thyroid cancer due to close monitoring of disease done by performing more frequent radiographic scans.

\section{CONCLUSIONS}

To our knowledge, pulmonary MAC has not previously been reported in association with any type of thyroid cancer. We present two interesting cases of octogenarian women with active Hürthle cell thyroid carcinoma who developed pulmonary MAC infection after prolonged periods of hypothyroidism. Further observations and studies are necessary to understand the potential relationship between the two diseases.

\section{REFERENCES}

[1] Watson, R., Brennan, M., Goellner, J., et al. (1984) Invasive Hürthle cell carcinoma of the thyroid: Natural his- 
tory and management. Mayo Clinic Proceedings, 59(12), 851-855.

[2] Grossman, R. and Clark, O. (1977) Hürthle cell carcinoma. Cancer Control, 4, 13-17.

[3] Yutan, E. and Clark, O. (2001) Hürthle cell carcinoma. Current Treatment Options in Oncology, 2(4), 331-335.

[4] Good, R. and Snider, D. (1982) Isolation of nontuberculous mycobacteria in the United States. Journal of Infectious Diseases, 146(6), 829-833.

[5] Pallella, F. Jr., Delaney, K., Moorman, A., et al. (1998) Declining morbidity and mortality among patients with advanced human immunodeficiency virus infection. HIV outpatient study investigators. New England Journal of Medicine, 338(13), 853-860.

[6] Parrish, S., Myers, J. and Lazarus, A. (2008) Nontuberculous mycobacterial pulmonary infections in non-HIV patients. Postgraduate Medicine, 120(4), 78-86.

[7] Erasmus, J., McAdams, H., Farrell, M. and Patz, E. Jr. (1999) Pulmonary nontuberculous mycobacterial infection: Radiologic manifestations. Radiographics, 19(6), 1487-1503.

[8] Levin, D. (2002) Radiology of pulmonary Mycobacterium avium-intracellulare complex. Clinics in Chest Medicine, 23(3), 603-612.

[9] Koh, W., Lee, K., Kwon, O., Jeong, Y., et al. (2005) Bilateral bronchiectasis and bronchiolitis at thin-section CT: Diagnostic implications in nontuberculous mycobacterial pulmonary infection. Radiology, 235(1), 282-288.

[10] Olivier, K., Weber, D., Lee, J., et al. (2003) Nontuberculous mycobacteria I: Multicenter prevalence study in cystic fibrosis. American Journal of Respiratory and Critical Care Medicine, 167(6), 828-834.

[11] Hartman, T., Jensen, E., Tazelaar, H., Hanak, V. and Ryu, J. (2007) CT findings of granulomatous pneumonitis secondary to Mycobacterium avium-intracellulare inhalation: "Hot tub lung." American Journal of Roentgenology, 188(4), 1050-1053.

[12] Reich, J. and Johnson, R. (1992) Mycobacterium avium complex pulmonary disease presenting as an isolated lingular or middle lobe pattern: The Lady Windermere syndrome. Chest, 101(6), 1605-1609.

[13] Prince, D., Peterson, D., Steiner, R., et al. (1989) Infection with Mycobacterium avium complex in patients without predisposing conditions. New England Journal of Medicine, 321(13), 863-868.

[14] Huang, J., Kao, P., Adi, V. and Ruoss, S. (1999) Mycobacterium avium-intracellulare pulmonary infection in HIV-negative patients without preexisting lung disease. Chest, 115(4), 1033-1040.

[15] Kim, R., Greenberg, D., Ehrmantraut, M., et al. (2008) Pulmonary nontuberculous mycobacterial disease. American Journal of Respiratory and Critical Care Medicine, 178(10), 1066-1074.

[16] Schoenfeld, P., Myers, J., Myers, L. and LaRocque, J. (1995) Suppression of cell-mediated immunity in hypothyroidism. Southern Medical Journal, 88(3), 347-349.

[17] Fabris, N. (1973) Immunodepression in thyroid-deprived animals. Clinical \& Experimental Immunology, 15(4), 601-611.

[18] Tani, H., Nabetani, T., Sasai, K. and Baba, E. (2005) Proliferative responses to canine thyroglobulin of peripheral blood mononuclear cells from hypothyroid dogs. Journal of Veterinary Medical Science, 67(4), 363-368.

[19] Mazzaferri, E. and Kloos, R. (2001) Current approaches to primary therapy for papillary and follicular cancer. Journal of Clinical Endocrinology \& Metabolism, 86(4), 1447-1463. 